\title{
New levels of sophistication in the transcriptional landscape of bacteria
}

\author{
Arnoud HM van Vliet* and Brendan W Wren ${ }^{\dagger}$
}

Addresses: *Institute of Food Research, Colney Lane, Norwich NR4 7UA, UK. †Department of Infectious and Tropical Diseases, London School of Hygiene and Tropical Medicine, Keppel Street, London WC1E 7HT, UK.

Correspondence: Brendan W Wren. Email: brendan.wren@Ishtm.ac.uk

\begin{abstract}
An extra layer of complexity in the regulation of gene expression in bacteria is now apparent through previously unanticipated roles of noncoding and antisense RNAs.
\end{abstract}

Bacteria are the great survivors on planet Earth, where they can adapt and flourish in harsh environments ranging from deep-sea vents to acidic mine shafts. A feature of many bacteria, particularly pathogenic bacteria, is their ability to adapt and thrive in multiple environments, which provides them with a competitive advantage. For example, the facultative intracellular pathogen Listeria monocytogenes happily survives in the ambient environment as a saprophyte, but on occasions it has an inherent capacity to turn nasty and cause brain and materno-fetal infections in humans [1]. This requires the bacterium to switch genes on and off as it traverses different environments, ranging from a saprophytic lifestyle to the gut lumen after ingestion to invasion of epithelial cells and intracellular survival. The key to the survivalist success of pathogens is their ability to coordinate, redirect and fine-tune their genetic repertoire as and when required. Traditionally, transcriptional reshaping in bacteria has been considered to be controlled by a hierarchical network of interconnected global transcriptional regulators, such as sigma factors and one- and two-component regulatory systems [2]. In the past decade it has become apparent that the various forms of noncoding regulatory RNA (previously considered as intergenic junk) play important roles in the global regulation of cellular functions, and may represent connecting links between many cellular networks $[3,4]$. As such, noncoding RNA also plays a subtle but crucial role in the coordination of the expression of bacterial virulence determinants [5]. Two recent papers from Pascale Cossart and colleagues [6,7] present a comprehensive microarray analysis of the transcriptome of Listeria monocytogenes in different conditions, uncovering an unsuspected variety of regulatory roles for noncoding RNAs in controlling changes in gene expression that characterize the transition from saprophytic to pathogenic lifestyle.

\section{Bacterial regulatory RNAs are more than intergenic junk}

As well as the familiar types of RNA - messenger RNA, ribosomal RNA, and transfer RNA - bacteria also express many other noncoding RNAs (Figure 1). Some of these are catalytic, such as RNase $\mathrm{P}$, or interact directly with proteins, like the $6 \mathrm{~S}$ RNA that interacts directly with $\sigma^{70_{-}}$ containing RNA polymerase [8] or the CsrA-sequestering CsrB and CsrC RNAs [9]. Most bacterial noncoding RNAs, however, are thought to have roles in the posttranscriptional regulation of gene expression, using their capacity for complementary base-pairing [3,4]. Regulatory RNAs are usually subdivided into different groups in respect to their genomic position: one group contains those encoded in cis with their target gene (such as riboswitches and antisense RNAs); the other those encoded in trans from their target gene, which are often located at completely unrelated positions on the genome, such as the canonical small RNAs (sRNA).

Cis-encoded regulatory RNAs in principle enable a multitude of regulatory responses to stimuli, but they are mostly used for the sensing of temperature, metabolites, or metabolic stimuli (Figure 1a). They can, for example, function in translational control as riboswitches [4], where the full-length mRNA is preceded by a folded 5' untranslated region (5' UTR), which can fold in different conformations depending on the stimulus, often either allowing or blocking access to the ShineDalgarno ribosome-binding sequence, thus controlling translation (Figure 1a). Examples are the prfA thermosensor of L. monocytogenes [10], which is involved in controlling virulence genes, or the cyclic-di-GMPsensing riboswitch of Vibrio cholerae [11], which controls biofilm formation, cell differentiation, and virulence gene expression. Alternatively, the 5' UTR can form transcriptional terminator or antiterminator loops (see Figure 1a), depending on the stimulus, and as such, control transcription of the downstream gene. An example of this is in the Escherichia coli tryptophan biosynthesis operon [12]. 


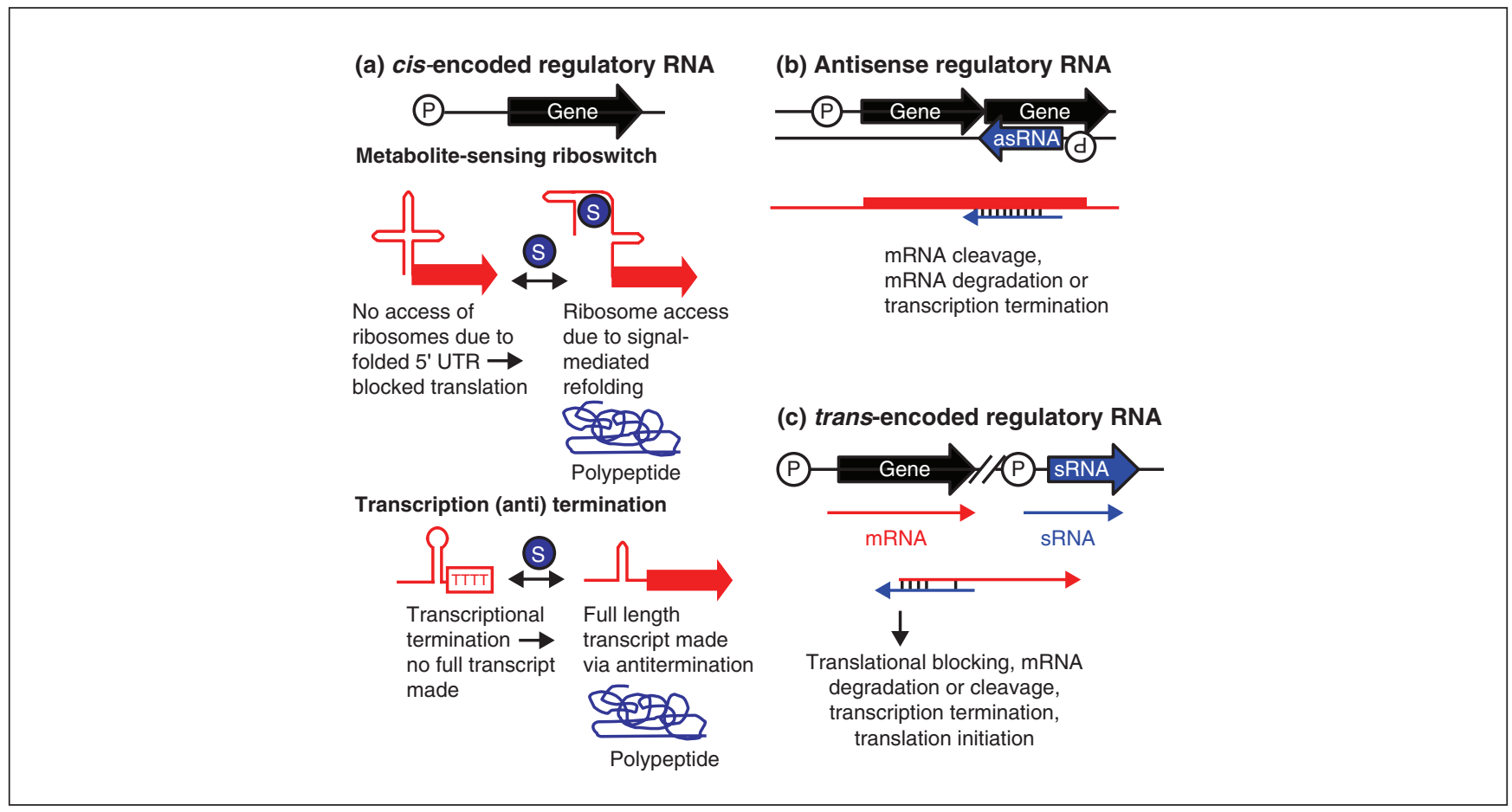

Figure 1

Schematic representation of the different modes of action of transcriptional regulation by noncoding RNA. (a) Regulation by cis-encoded RNA, subdivided into signal-mediated folding or unfolding of the translation initiation region (top) or transcription termination/antitermination (bottom). (b) Regulation by antisense RNA (asRNA). (c) Regulation by trans-encoded sRNA. Circled P, promoter; circled S, environmental or metabolic signal.

A relatively newly discovered class of cis-encoded regulatory RNA is antisense RNA (Figure 1b). Antisense RNA is known to be involved in bacterial Type I toxin-antitoxin systems [13] and the control of incompatibility of some plasmids [14], but has not been implicated in controlling the expression of virulence genes. However, this may be because microarrays used in functional genomics and transcriptome studies have not incorporated probes for potential antisense transcripts. Antisense RNA shows perfect complementarity to its target sequences, and can either silence gene expression or lead to alternative processing of the mRNA.

Trans-encoded regulatory RNAs are the best-known class of noncoding RNA (Figure 1c) and represent the prokaryotic version of RNA interference [15]. Bacterial sRNAs are anywhere between 30 and 500 nucleotides in length, but the complementary base-pairing is often restricted to a small aptamer of 8-20 nucleotides, which may have imperfect complementarity to its target mRNAs [3]. The effects of base-pairing range from translational blocking (when bound to the 5' UTR overlapping the ShineDalgarno sequence) to mRNA degradation, although there are also examples where sRNA binding induces translation of the target mRNA [3,16]. Most information on bacterial
sRNA regulation has been obtained from analysis of $E$. coli and Salmonella enterica serovar Typhimurium, in which sRNA regulation controls many important aspects of cellular responses, ranging from outer membrane protein composition to metabolism [5].

\section{Comprehensive transcriptome analysis and transcriptional reshaping}

While the complexity of eukaryotic transcriptional regulation is well appreciated [17], it was long thought that bacteria lacked many of the sophisticated regulatory mechanisms affecting the cellular transcription landscape. However, recent papers have shed new light on this subject, and have revealed that notions of the relative simplicity of bacterial gene expression severely underestimates the potential of bacteria to control expression of their genetic repertoire [6,18-21].

Pascale Cossart and colleagues (Toledo-Arana et al. [6]) present one of the first comprehensive unbiased transcriptomes of a bacterium, in this case L. monocytogenes, that includes noncoding and antisense RNAs. This tour de force reveals that gene regulatory mechanisms in bacteria are far more complex than previously appreciated. Using an unbiased, high-density tiling microarray consisting of 
345,668 25-base oligonucleotide probes covering the whole genome, they have shown antisense RNAs spanning several open reading frames and long overlapping 5 ' and 3 ' UTRs, in addition to 50 sRNAs. One surprising finding was the presence of long 5' UTRs which functioned as antisense RNA, as observed upstream of the $\operatorname{mog} R$ gene encoding a flagellar regulatory protein [6]. By comparing transcripts from bacteria grown in different physiological conditions (hypoxia, stationary phase, and low temperature) and from different in vivo conditions (intestinal lumen and blood), at least two of the sRNAs were found to be involved in virulence. One of these was rli38, which was upregulated 25-fold in blood and can pair to three mRNAs including that for fur, which encodes a global iron-uptake regulator. A subsequently constructed rli38 mutant was attenuated in the murine listeriosis infection model. Significantly, rli38 is absent from Listeria innocua, a nonpathogenic species closely related to $L$. monocytogenes. To assess transcriptional reshaping further, Toledo-Arana et al. [6] also investigated the transcriptomes of bacteria mutant for the global regulators PrfA (a virulence determinant regulator), SigB (an alternative sigma factor) and Hfq (an RNA chaperone). Together, these approaches identified the differential regulation of several additional RNA elements including cis-regulatory RNAs and overlapping UTRs, providing a comprehensive picture of how the transcriptome changes as the microorganism cycles through saprophytism and virulence. The results of the in vivo transcriptional profiling were largely verified in a study by Camejo et al. [7] using a L. monocytogenes macroarray.

Whereas the microarray studies described above give unique insights in the biology of L. monocytogenes (as did previous tiling microarray studies on E. coli [22] and Caulobacter crescentus [23]), we may have now started to reach the limits of what microarray and other hybridization-based techniques can tell us [24]. Microarrays have a relatively limited dynamic range for the detection of transcript levels, owing to background, cross-hybridization, saturation and spot density and quality, and require a complete genome sequence for probe design. Also, comparison of transcription levels between independent microarray experiments is rather challenging and mostly based on complex normalization methods [25]. Finally, microarray technology only measures the relative level of RNA, and does not distinguish between de novo synthesized transcripts and modified transcripts; nor does it allow accurate determination of the promoter used in the case of $d e$ novo transcription. Many of these issues can now be resolved by high-throughput sequencing of cDNA libraries [24].

This is illustrated in recent work by Yoder-Himes et al. [18], who used high-throughput sequencing to compare transcriptional patterns between two Burkholderia cenocepacia strains in conditions mimicking cystic fibrosis (CF) and soil, using strains originally isolated from a patient with $\mathrm{CF}$ and from soil. They also report significant changes in the transcription of noncoding RNA that are important in adaptation to external conditions. Other researchers have described transcriptomics or noncoding RNA identification using high-throughput sequencing in the bacterial pathogens Bacillus anthracis and V. cholerae $[19,20]$, while alternative approaches, such as immunoprecipitation of nucleic-acid-binding proteins, have been used to identify RNAs bound to the RNA chaperone Hfq in Salmonella [21].

Many regulatory noncoding RNAs were first described in E. coli and related Enterobacteriaceae, and depend on Hfq for their mode of action. However, with the advent of highdensity microarrays and high-throughput sequencing, it is clear that paradigms based on $E$. coli do not necessarily hold true in other bacteria. Some bacteria lack Hfq and can be predicted to use alternative mechanisms for RNA regulation, while in many Hfq-positive bacteria the role of Hfq is still unclear [16]. Other RNA-binding proteins, such as CsrA, may play important roles beyond their originally described functions; in the case of CsrA this is binding to Shine-Dalgarno-like sequences [9]. All these regulatory pathways taken together allow for fine-tuning of the cellular transcriptional mechanisms, giving the bacterium the best of options to survive in adverse conditions.

It is now clear that, as in eukaryotes, bacteria exploit noncoding RNAs in their genetic regulatory repertoire, destroying another myth in the distinction between eukaryotes and prokaryotes. The development of 'next generation' DNA sequencing and direct RNA sequencing will no doubt throw even more light on the role of RNA in gene regulation $[26,27]$. This will pave the way to a new era in understanding the complex and dynamic mechanisms by which bacteria adapt to the multiple environments they encounter. The next few years promise to be a voyage of discovery in terms of understanding the previously underestimated role of RNA in bacterial gene regulation.

\section{Acknowledgements}

AvV is supported by the BBSRC Institute Strategic Programme Grant to the IFR. BWW is supported by the BBSRC and The Wellcome Trust.

\section{References}

1. Cossart P, Toledo-Arana A: Listeria monocytogenes, a unique model in infection biology: an overview. Microbes Infect 2008, 10:1041-1050.

2. Bijlsma JJ, Groisman EA: Making informed decisions: regulatory interactions between two-component systems. Trends Microbiol 2003, 11:359-366.

3. Waters LS, Storz G: Regulatory RNAs in bacteria. Cell 2009, 136:615-628.

4. Winkler WC, Breaker RR: Regulation of bacterial gene expression by riboswitches. Annu Rev Microbiol 2005, 59: 487-517.

5. Vogel J: A rough guide to the noncoding RNA world of Salmonella. Mol Microbiol 2008, 71:1-11. 
6. Toledo-Arana A, Dussurget O, Nikitas G, Sesto N, Guet-Revillet $\mathrm{H}$, Balestrino D, Loh E, Gripenland J, Tiensuu T, Vaitkevicius K, Barthelemy $M$, Vergassola $M$, Nahori MA, Soubigou G, Régnault B, Coppée JY, Lecuit M, Johansson J, Cossart P: The Listeria transcriptional landscape from saprophytism to virulence. Nature 2009, 459:950-956.

7. Camejo A, Buchrieser C, Couve E, Carvalho F, Reis O, Ferreira $\mathrm{P}$, Sousa $\mathrm{S}$, Cossart $\mathrm{P}$, Cabanes $\mathrm{D}$ : In vivo transcriptional profiling of Listeria monocytogenes and mutagenesis identify new virulence factors involved in infection. PLOS Pathog 2009, 5:e1000449.

8. Wassarman KM: 6S RNA: a regulator of transcription. Mol Microbiol 2007, 65:1425-1431.

9. Lucchetti-Miganeh C, Burrowes E, Baysse C, Ermel G: The post-transcriptional regulator CsrA plays a central role in the adaptation of bacterial pathogens to different stages of infection in animal hosts. Microbiology 2008, 154:16-29.

10. Narberhaus F, Waldminghaus T, Chowdhury S: RNA thermometers. FEMS Microbiol Rev 2006, 30:3-16.

11. Sudarsan N, Lee ER, Weinberg Z, Moy RH, Kim JN, Link KH, Breaker RR: Riboswitches in eubacteria sense the second messenger cyclic di-GMP. Science 2008, 321:411-413.

12. Yanofsky C: The different roles of tryptophan transfer RNA in regulating trp operon expression in $E$. coli versus $B$. subtilis. Trends Genet 2004, 20:367-374.

13. Van Melderen L, Saavedra De Bast M: Bacterial toxin-antitoxin systems: more than selfish entities? PLOS Genet 2009, 5:e1000437.

14. MacLellan SR, Smallbone LA, Sibley CD, Finan TM: The expression of a novel antisense gene mediates incompatibility within the large repABC family of alpha-proteobacterial plasmids. Mol Microbiol 2005, 55:611-623.

15. Mattick JS: The genetic signatures of noncoding RNAs. PLoS Genet 2009, 5:e1000459.

16. Valentin-Hansen $P$, Eriksen $M$, Udesen $C$ : The bacterial Sm-like protein Hfq: a key player in RNA transactions. Mol Microbiol 2004, 51:1525-1533.

17. Cloonan N, Grimmond SM: Transcriptome content and dynamics at single-nucleotide resolution. Genome Biol 2008, 9:234

18. Yoder-Himes DR, Chain PS, Zhu Y, Wurtzel O, Rubin EM, Tiedje JM, Sorek R: Mapping the Burkholderia cenocepacia niche response via high-throughput sequencing. Proc Natl Acad Sci USA 2009, 106:3976-3981.
19. Liu JM, Livny J, Lawrence MS, Kimball MD, Waldor MK, Camilli A: Experimental discovery of sRNAs in Vibrio cholerae by direct cloning, 5S/tRNA depletion and parallel sequencing. Nucleic Acids Res 2009, 37:e46.

20. Passalacqua KD, Varadarajan A, Ondov BD, Okou DT, Zwick $\mathrm{ME}$, Bergman $\mathrm{NH}$ : Structure and complexity of a bacterial transcriptome. J Bacteriol 2009, 191:3203-3211.

21. Sittka A, Lucchini S, Papenfort K, Sharma CM, Rolle K, Binnewies TT, Hinton JC, Vogel J: Deep sequencing analysis of small noncoding RNA and mRNA targets of the global post-transcriptional regulator, Hfq. PLOS Genet 2008, 4: e1000163.

22. Selinger DW, Cheung KJ, Mei R, Johansson EM, Richmond CS, Blattner FR, Lockhart DJ, Church GM: RNA expression analysis using a $\mathbf{3 0}$ base pair resolution Escherichia coli genome array. Nat Biotechnol 2000, 18:1262-1268.

23. McGrath PT, Lee H, Zhang L, Iniesta AA, Hottes AK, Tan MH, Hillson NJ, Hu P, Shapiro L, McAdams HH: High-throughput identification of transcription start sites, conserved promoter motifs and predicted regulons. Nat Biotechnol 2007, 25:584-592

24. Hoen PAT, Ariyurek $\mathrm{Y}$, Thygesen $\mathrm{HH}$, Vreugdenhil E, Vossen $\mathrm{RH}$, de Menezes RX, Boer JM, van Ommen GJ, den Dunnen JT: Deep sequencing-based expression analysis shows major advances in robustness, resolution and inter-lab portability over five microarray platforms. Nucleic Acids Res 2008, 36:e141.

25. Hinton JC, Hautefort I, Eriksson S, Thompson A, Rhen M Benefits and pitfalls of using microarrays to monitor bacterial gene expression during infection. Curr Opin Microbiol 2004, 7:277-282.

26. MacLean D, Jones JDG, Studholme DJ: Application of 'nextgeneration' sequencing technologies to microbial genetics. Nat Rev Microbiol 2009, 7:287-296.

27. Morris DR: Ribosomal footprints on a transcriptome landscape. Genome Biol 2009, 10:215.

Published: 3 August 2009

doi:10.1186/gb-2009-10-8-233

(c) 2009 BioMed Central Ltd 